\title{
Thöne, Meik, Autonome Systeme und deliktische Haftung.
}

\author{
Mohr Siebeck Verlag, Tübingen, 2020. 311 Seiten. ISBN \\ 978-3-16-159492-2
}

\section{Christian Armbrüster}

Online publiziert: 16. Juli 2020

(C) Der/die Autor(en) 2020

Die fortschreitende Digitalisierung bringt für die Deliktshaftung und damit auch für die Haftpflichtversicherung vielfältige Herausforderungen mit sich. Dies wird besonders deutlich im Bereich autonomer Systeme, die bestimmte, bislang von Menschen ausgeführte Tätigkeiten eigenständig ausführen. Prominente Beispiele sind autonome Fahrzeuge, Operations- und Pflegeroboter oder Drohnen. Ein Haftungsrecht, das wie das deutsche für die Verpflichtung zum Schadensersatz in erster Linie an ein schuldhaftes menschliches Verhalten anknüpft, wird durch autonome Systeme mithin vor die Frage gestellt, wie ,zukunftsfähig“ diese Anknüpfung noch ist. Als Alternativen werden insbesondere eine weit reichende verschuldensunabhängige Gefährdungshaftung, eine Verlagerung der Haftung weg von denjenigen Personen, die autonomen Systeme einsetzen, und hin zu den Produzenten der Hard- und Software dieser Systeme sowie die Haftungsersetzung durch umlagen- oder steuerfinanzierte Fondsmodelle diskutiert.

Der Autor der vorliegenden Neuerscheinung geht einen anderen Weg. Er kündigt bereits im Vorwort an, dass es ihm darum geht, ,Bestehendes zunächst anzupassen statt es allzu voreilig Neuem zu opfern“. Dieser Ansatz weckt auch aus versicherungsrechtlicher Sicht das Interesse des Lesers. In der aktuellen Debatte um die künftige Haftungs- und Versicherungsregeln beim autonomen Fahren geht es nämlich insbesondere darum, inwiefern sich die hergebrachte Kombination einer verschuldensunabhängigen Halterhaftung mit einer Pflicht-Haftpflichtversicherung des Halters im Bereich der Kfz-Unfälle aufrechterhalten und womöglich auf andere Bereiche, in denen gegenwärtig weder eine verschuldensunabhängige Haftung noch eine Versicherungspflicht besteht, erstrecken lässt (s. dazu die Dokumentation des Karlsruher Forums 2020, das sich intensiv dem von Thöne thematisierten Fragen-

C. Armbrüster $(\bowtie)$

Fachbereich Rechtswissenschaft, Freie Universität Berlin, Van't-Hoff-St. 8, 14195 Berlin,

Deutschland

E-Mail: c.armbruester@fu-berlin.de 
kreis gewidmet hat, in VersR 2020, $717 \mathrm{ff}$., mit den Vorträgen von G. Wagner und $R$. Koch sowie einem ausführlichen Diskussionsbericht). Man ist also gespannt zu erfahren, in welcher Weise der Verfasser seinen angekündigten behutsamen Ansatz konkretisiert.

Die erste Hälfte seines Werks widmet Thöne einer ausführlichen Grundlegung (S. 25-153). Dabei bietet er einen instruktiven Überblick zum gegenwärtigen Stand der Verschuldens- und der Gefährdungshaftung im deutschen Recht. Zutreffend stellt er eine Tendenz des modernen Haftungsrechts hin zu einer Ausweitung der Gefährdungshaftung fest (S. 145 ff.). Dabei verweist er zu Recht auch auf die europäischen Bestrebungen in dieser Richtung. Ein anschauliches Beispiel ist etwa die in der Umwelthaftungsrichtlinie 2004/35/EG vorgegebene und in Deutschland im Umweltschadensgesetz von 2007 umgesetzte weitgehend verschuldensunabhängige Haftung der Verursacher von Umweltschäden (näher dazu Armbrüster/Schreier, ZVersWiss 2016, 3 ff.). Im Bereich der Haftung für Robotik liegen auf EU-Ebene mittlerweile Vorschläge vor, die insgesamt - wie der Autor (S. 148) zu Recht hervorhebt - keine substanzielle Abkehr vom Verschuldensprinzip bedeuten. Instruktiv sind auch die Ausführungen zur wechselseitigen Annäherung von Verschuldensund Gefährdungshaftung (S. $151 \mathrm{ff}$.); diese treten im Bereich der Produkt- und der Produzentenhaftung besonders deutlich hervor (S. $121 \mathrm{ff}$.).

Plastisch arbeitet Thöne sodann die Herausforderungen heraus, vor die autonome Systeme das hergebrachte Haftungsrecht stellen. Hier ist an erster Stelle das komplizierte Geflecht der Verantwortlichkeiten zu nennen (S. $161 \mathrm{ff}$.). Dabei sind nicht allein Inhaber (Halter, Eigentümer), Nutzer und Hersteller sowie deren Zulieferer betroffen, sondern - etwa bei autonomen Fahrzeugen - auch die gesamte digitale Umgebungs-Infrastruktur, deren ordnungsgemäßes Funktionieren für einen schadenfreien Verlauf entscheidend sein kann. Unter der Überschrift ,dogmatische Überlegungen“ (S. 167) unternimmt Thöne es, den Ausnahmecharakter der Gefährdungshaftung anhand einzelner Regelungen des BGB zu belegen. Die Einführung einer Gefährdungshaftung sei daher rechtfertigungsbedürftig, und es müsse bei der Verschuldenshaftung bleiben, sofern sich kein ,hinreichender Grund“ (S. 168) dafür anführen lasse, eine strengere Haftung zu statuieren. Diesem Befund vermag man ohne Weiteres zu folgen; freilich folgt das Erfordernis, dass eine Gefährdungshaftung der Rechtfertigung bedarf, verfassungsrechtlich bereits aus der damit verbundenen Einschränkung der allgemeinen Handlungsfreiheit (Art. 2 Abs. 1 GG) und ggf. der Berufsausübungsfreiheit (Art. 12 Abs. 1 GG) sowie aus dem Verhältnismäßigkeitsprinzip. Der nachfolgende Abschnitt ist ökonomischen Überlegungen gewidmet; er führt den Autor zu dem Befund, dass die ökonomische Analyse jedenfalls keine zwingenden Gründe für eine Gefährdungshaftung zu bieten vermag (S. 180). Dies verdient Zustimmung. Auch die Ausführungen dazu, warum eine ePerson als neues Haftungssubjekt abzulehnen ist, überzeugen. Die Einwände gegen diese neue Rechtsfigur sind schlagend: Zum einen würde sie ohne eigene Vermögensausstattung von vornherein nicht als Haftungssubjekt taugen. Mit guten Grund ist der früher im englischen Common Law geltende Grundsatz, dass eine Sache, die einen Schaden verursacht hat, zugunsten des Geschädigten verwertet werden kann (sog. deodand), im 19. Jhd. zugunsten einer Kombination von Gefährdungshaftung und Versicherung aufgegeben worden (Tasioulas, Journal of Practical Ethics 2019, 87, $108 \mathrm{f}$.). 
Zum anderen würde die Einführung einer ePerson es den Nutzern und Herstellern autonomer Systeme ermöglichen sich der Verantwortung zu entziehen; damit würde auch eine Verhaltenssteuerung - auf die Thöne freilich nicht näher eingeht - vereitelt (S. 182f.).

Von besonderem Interesse sind aus versicherungsrechtlicher Sicht die Vorschläge, die der Autor für die Haftung unterbreitet. Sie laufen im Kern darauf hinaus, dass er auf der Grundlage der von ihm favorisierten Verschuldenshaftung - spezifische Verkehrspflichten herausarbeitet (S. 195ff.). Diese Pflichten betreffen Technik und Daten, Systemveränderlichkeit und „Herstellerkontrolle“ (gemeint ist: Produktkontrolle durch den Hersteller), Beobachtung und Wartung, Systemeingriffe und Vernetzungsrisiken, Sicherheitsmodus und Steuerungsübernahme, Mensch-Maschine-Interaktion und Nutzerinstruktion sowie Kommunikation und „,bewussten Rechtsbruch“ (etwa durch Verständigung von autonomen Fahrzeugen in unklaren Verkehrssituationen). Die konkreten Verhaltensanforderungen, die Thöne jeweils zumindest kurz umreißt, erinnern teils an die Obliegenheiten, die in der Cyberrisiko-Versicherung nach den meisten AVB den Versicherungsnehmer treffen. Insofern vermag das Buch durchaus auch Anregungen dafür zu bieten, entsprechende Obliegenheitskataloge in konkreten AVB daraufhin zu überprüfen, ob sie die von Thöne genannten Aspekte hinreichend abdecken.

Interessanterweise möchte der Autor trotz seines nachdrücklichen Plädoyers für eine verschuldensabhängige Haftung hinsichtlich autonomer Fahrzeuge an der Halterhaftung im Straßenverkehr festhalten. Er begründet dies mit der Kontinuität, vor allem aber mit dem Opferschutz. Zugleich meint er, die Gefährdungshaftung solle außer dem Nutzer (sic; es bleibt im Dunkeln, wieso es trotz der angestrebten Kontinuität der Haftungsregeln beim Auseinanderfallen von Nutzer und Halter nicht entscheidend auf den Halter ankommen soll) auch den Hersteller ,adressieren“ (S. 243), da eine einseitige Haftungsbelastung nicht dem Kontroll- und Einwirkungsvermögen der „Protagonisten“ (S. 244) entspräche. Nutzer und Hersteller bildeten dann einen Haftungsverbund ( $\S 840$ Abs. 1, 421 ff. BGB). Dieser Verbund könne durch beiderseitige Pflichtversicherungen und entsprechende „Abrechnungsabkommen“ der Versicherer ergänzt werden (S. 244).

Das solchermaßen mit wenigen Worten und ohne nähere Analyse der Interessenlage skizzierte Haftungs- und Pflichtversicherungsregime wirft zahlreiche Fragen auf. So geht Thöne nicht näher darauf ein, dass sub specie des Opferschutzes die Halterhaftung völlig ausreichend ist und nicht der Flankierung durch eine (offenbar sektorenspezifische, nur Kfz-Hersteller treffende) Gefährdungshaftung des Herstellers bedarf. Bereits nach geltendem Recht ist der Versicherer aufgrund der Legalzession nach $\S 86$ Abs. 1 VVG in der Lage, den Hersteller auf der Grundlage der Produktund Produzentenhaftung in Regress zu nehmen. Da es sich bei der Kfz-Haftpflichtversicherung um eine Pflichtversicherung handelt, wird der Halter ohnehin von einer persönlichen Einstandspflicht - sieht man einmal von den wirtschaftlichen Nachteilen ab, die mit einer Rückstufung für die Prämienhöhe verbunden sind - entlastet. Das Argument, Nutzer und Hersteller müssten gleichermaßen belastet werden, büßt dadurch entscheidend an Überzeugungskraft ein.

Mit dem Vorschlag, auch für den Hersteller eine Gefährdungshaftung sowie eine Versicherungspflicht einzuführen, entfernt sich der Autor auch von seinem bereits 
erwähnten, hinsichtlich der Gefährdungshaftung aufgestellten Postulat, für diese sei stets ein „hinreichender Grund“ (S. 168) erforderlich. Gerade die Einführung neuer Pflichtversicherungen sollte nur erfolgen, wenn dies im Interesse des Opferschutzes unabweisbar geboten ist. Angesichts der vorhandenen umfassenden Regeln zum Verkehrsopferschutz ist dies nicht der Fall. So bietet etwa das System der Grünen Karte dem Opfer einen effizienten Schutz, der selbst dann eingreift, wenn der Halter nicht versichert oder nicht identifizierbar ist. Für Kfz-Hersteller fehlen entsprechende Regeln; sie sind angesichts des bewährten Kfz-Versicherungsregimes auch nicht erforderlich (s. dazu den Tagungsbericht von Biller-Bomhardt/Kunz, VersR 2020, 755, 759). Letztlich verliert Thöne in diesem Kontext einen grundlegenden Befund aus dem Blick, der sein Bemühen um eine Einbeziehung beider von ihm sog. ,Protagonisten“, nämlich Nutzer und Hersteller, wesentlich relativiert: Letztlich hat für die Kosten des Haftungs- und Versicherungsschutzes stets der Halter aufzukommen, sei es über die Kfz-Versicherungsprämie oder über den Preis für das Kfz. Dieser Befund führt dazu, dass die Frage nach der sinnvollen Haftungsallokation letztlich allein anhand des Kriteriums zu beantworten ist, welche Lösung für das Opfer am günstigsten ist. Hier hat die Kombination aus Halterhaftung und Pflicht-Haftpflichtversicherung nach wie vor die besseren Argumente für sich (näher Armbrüster, in: Festschrift für Christian Huber, 2020, S. 27 ff.; s. auch dens., LMCLQ 2020, Part 1, February 2020, p. 86-103).

Von seiner grundsätzlichen Positionierung zugunsten der Verschuldenshaftung aus ist es konsequent, dass Thöne sich nicht näher mit Überlegungen auseinandersetzt, das Haftungs- und Versicherungskonzept aus dem Kfz-Bereich auf andere autonome Systeme als autonome $\mathrm{Kfz} z u$ übertragen. Eine solche Lösung könnte sich zumindest für autonome Transportsysteme, etwa mit Drohnen, anbieten. Immerhin kommt er ganz am Ende seiner Untersuchung noch einmal gesondert kurz auf die Pflichtversicherung zu sprechen (S. 261-263). Dabei spricht er sich im Grundsatz gegen eine umfassende Versicherungspflicht aus; vielmehr solle eine solche Pflicht „,auf besonders gefahrenträchtige Bereiche begrenzt sein“ (S. 262), in denen ein potentiell Haftpflichtiger wohl nicht in der Lage sein werde sämtliche Schäden auszugleichen. Thöne plädiert weiter dafür, im Rahmen neu zu schaffender Pflichtversicherungen auch die Schutzinstrumente einführen, die im Bereich der Kfz-Pflichtversicherung bestehen. Dies wird man hinsichtlich der Schaffung eines umlagefinanzierten Entschädigungsfonds nach dem Vorbild von $§ 12$ PflVG durchaus in Erwägung ziehen können. Schwieriger dürfte es werden, im Bereich jenseits von Kfz dem Geschädigten eine leichte Identifikation des jeweiligen Haftpflichtversicherers zu ermöglichen. Man müsste dann wohl für den Halter bzw. Nutzer des jeweiligen schadenstiftenden autonomen Systems eine Registrierungspflicht einführen, mittels derer sich der Haftpflichtversicherer über eine Auskunftsstelle ermitteln lässt. Der von Thöne gebildete Anwendungsfall einer solchen Fondslösung, dass aus der Auslesung des Datenrekorders kein Schuldvorwurf dokumentiert oder Kausalitätsnachweis erbracht werden kann, erscheint auf der Grundlage des von ihm vertretenen Konzepts einer verschuldensabhängigen Haftung konsequent; geht man hingegen von einer Halterhaftung aus, so kommt es von vornherein allein auf die (Mit-) Verursachung an, während die Schuldfrage nicht klärungsbedürftig ist. Letz- 
teres ist aus Sicht der Geschädigten im geltenden Kfz-Haftungsrecht ein nicht zu unterschätzender Vorteil.

$\mathrm{Ob}$ es nicht konsequent erscheint, diesen Vorteil künftig auch denjenigen zugute kommen zu lassen, die von einem autonomen System geschädigt werden, und den Schaden verschuldensunabhängig demjenigen aufzuerlegen, der sich dieses System als Halter zunutze macht und der zugleich durch eine Pflichtversicherung vor den Haftungsfolgen geschützt wird, ist eine Frage, auf die das Buch letztlich keine befriedigende Antwort gibt. Dies gilt umso mehr, als sich - wie die fließenden Übergänge bei der Produkt- und Produzentenhaftung zeigen (s. S. 123 mit Fn. 51) - nahezu jede Gefährdungshaftung letztlich auch auf die Verletzung einer Verkehrssicherungspflicht stützen ließe, dehnte man letztere nur weit genug aus.

Fazit Insgesamt bietet das flüssig geschriebene und mit einem reichhaltigen Fußnotenapparat versehene Buch eine anregende Lektüre auf aktuellem Stand. Eine in sich schlüssige Gesamtlösung für die Haftung und Versicherung bei autonomen Systemen wird darin freilich nicht geboten. Der schwerwiegendste Einwand dürfte aus systematischen und dogmatischen Gründen derjenige sein, dass Thöne das von ihm bevorzugte Modell der Verschuldenshaftung selbst nicht auf den wohl wichtigsten künftigen Anwendungsfall, nämlich die Haftung und Versicherung beim Einsatz autonomer Kfz, anwenden möchte. Die darin liegende Ungleichbehandlung verschiedener Erscheinungsformen autonomer Systeme vermag nicht ohne Weiteres zu überzeugen. Man darf gespannt sein, wie diese Diskussion sich weiter entwickeln wird.

Funding Open Access funding provided by Projekt DEAL.

Open Access Dieser Artikel wird unter der Creative Commons Namensnennung 4.0 International Lizenz veröffentlicht, welche die Nutzung, Vervielfältigung, Bearbeitung, Verbreitung und Wiedergabe in jeglichem Medium und Format erlaubt, sofern Sie den/die ursprünglichen Autor(en) und die Quelle ordnungsgemäß nennen, einen Link zur Creative Commons Lizenz beifügen und angeben, ob Änderungen vorgenommen wurden.

Die in diesem Artikel enthaltenen Bilder und sonstiges Drittmaterial unterliegen ebenfalls der genannten Creative Commons Lizenz, sofern sich aus der Abbildungslegende nichts anderes ergibt. Sofern das betreffende Material nicht unter der genannten Creative Commons Lizenz steht und die betreffende Handlung nicht nach gesetzlichen Vorschriften erlaubt ist, ist für die oben aufgeführten Weiterverwendungen des Materials die Einwilligung des jeweiligen Rechteinhabers einzuholen.

Weitere Details zur Lizenz entnehmen Sie bitte der Lizenzinformation auf http://creativecommons.org/ licenses/by/4.0/deed.de. 